\title{
DES CARÊMES APRÈS LE CARÊME STRATÉGIES DE CONVERSION ET FONCTIONS POLITIQUES DES MISSIONS INTÉRIEURES EN ESPAGNE ET AU PORTUGAL (1540-1650)
}

\author{
Marie-Lucie Copete et Federico Palomo
}

RésumÉ : L'absence de travaux sur les missions intérieures jésuites en Espagne et au Portugal ne correspond pas à l'abondance des documents qui attestent une forte activité missionnaire depuis la deuxième moitié du xvI ${ }^{\mathrm{e}}$ siècle. Le caractère fondamentalement quadragésimal de l'activité initiale des jésuites déterminera le sens pénitentiel des missions ibériques et, par conséquent, les stratégies missionnaires elles-mêmes. Les formes de « présentation » des jésuites et le genre de prédication qu'ils adressent aux fidèles sont des exemples de ces stratégies, vouées à l'objectif final, propres aux missions pénitentielles, de mener les fidèles à la "conversion ". Toutefois, cette idée de conversion prend un sens politique, qui trouve sa manifestation la plus évidente dans l'activité de pacification des communautés. L'action accomplie par les jésuites auprès des saisonniers dans les madragues de Conil et de Zahara en Andalousie montre le rôle joué par la mission intérieure comme moyen d'articulation du corps social, selon les modèles de comportement et d'organisation politique de l'époque.

Mots-CLÉs : missions intérieures, Espagne, Portugal, histoire religieuse, histoire politique, xvI'-xvII siècles.

ABsTRACT : The paucity of works on internal Jesuit missions in Spain and Portugal does not correspond to the abundance of accounts that attest to a strong activity from the second half of xvith century. The fundamentally quadragesimal character of the initial activity in this field would determine the penitential sense of the Iberian missions, and, as a consequence, the missionary strategies themselves. The " self-presentation " of Jesuits and the type of preaching that they made to the faithful are examples of such strategies, devoted to the ultimate objective of the penitential missions to lead the people to "conversion". In any case, this idea of conversion takes on a political sense, which is demonstrated most evidently in their peace-making activity. In this regard, the action undertaken by Jesuits in the Almadrabas of Conil and Zahara demonstrates the role played by internal missions with regard to the means of articulation of society, according to the patterns of behaviour and the models of political organization of this period.

KEYWORDS : internal missions, Spain, Portugal, religious history, political history, xvth-xvith centuries.

Revue de synthèse : $4^{\text {e }}$ S. $n^{\text {os }}$ 2-3, avr.-sept. 1999, p. 359-380. 
Zusammenfassung : Der Mangel an Arbeiten über die innere Missionierung in Spanien und Portugal seitens der Jesuiten steht in keinem Verhältnis zu den zahlreichen Zeitzeugen, die über eine starke Missionierungsaktivität seit der zweiten Hälfte des 16. Jahrhunderts berichten. Die missionarischen Tätigkeiten sind hauptsächlich in den Karwochen durchgeführt worden. Dadurch erhielten sie ihren reumütigen Charakter, und dadurch bestimmte sich auch die Missionierungsstrategie selbst. Die Darstellungsformen der gläubigen Jesuiten und die Art der Predigt, mit der sie sich an die Gläubigen wandten sind Beispiele solcher Strategien, die darauf abzielten, durch Reue die Gläubigen zur Umkehr zu bewegen. Jedoch beinhaltet die Idee der Umkehr auch eine politische Komponente, die sich vor allem in den Pazifizierungsaktivitäten niederschlägt. In dieser Hinsicht zeigen die Aktivitäten, die die Jesuiten in den Almadrabas von Conil und Zahara unternommen haben und unterstreichen in der Tat die Rolle der inneren Missionierung als Mittel sozialer Artikulation, gemä $\beta$ den Verhaltensregeln und den Modellen politischer Organisation jener Zeit.

STICHWÖRTER: innere Missionierung, Spanien, Portugal, Religionsgeschichte, politische Geschichte, 16. und 17. Jahrhunderte.

RESUMEN : La falta de estudios sobre las misiones interiores jesuitas en España y en Portugal es contradictoria con la abundancia de documentos conservados que indian muy al contrario una actividad misionera intensa en la segunda mitad del siglo xv. La característica fundamentalmente cuaresmal de la primera actividad de los jesuitas determina la orientación penitencial de las misiones ibéricas y de las mismas estrategias misioneras. Los modos de "presentación " de los misioneros y el tipo de predicación dirigido a los fieles son unos ejemplos de estas estrategias, gobernadas por la ambición final de toda mision penitencial : la conversión. Pero aquí la conversión toma un sentido político, cuya manifestación más clara es la tarea de pacificación de las comunidades. La acción desarrollada por los jesuitas entre los trabajadores estacionales de las madragas de Conil y Zahara, en Andalucía, demuestra la importancia de las misiones interiores como medio de articulación social, según los modelos de comportamiento y las formas de organización política de su tiempo.

Palabras Claves: misiones interiores, España, Portugal, historia religiosa y política, s. xV y xVu.

Marie-Lucie CoPEre, maître de conférences à l'université Nancy II et membre du Groupe de recherches sur les missions ibériques à l'époque moderne de l'EHESS, a soutenu, en 1995, une thèse à l'Institut universitaire européen de Florence sur les prisons et la pauvreté dans l'Espagne de la fin du xvi siècle.

Adresse : Université Nancy II, campus Lettres et Sciences humaines, BP 33-97, F-54015 Nancy. Courrier électronique : mlcopete@wanadoo.fr

Federico Palomo a étudié à Madrid et à l'Institut universitaire européen. Il est spécialiste de l'histoire religieuse de la Contre-Réforme dans la péninsule ibérique et, dans le cadre de sa thèse, a plus particulièrement travaillé sur les missions intérieures au Portugal entre 1550 et 1650.

Adresse : Institut universitaire européen, Badia Fiesolana, via dei Roccettini, 9, I-50016 San Domenico di Fiesole.

Courrier électronique : palomo@datacomm.iue.it 
La péninsule ibérique des $\mathrm{XVI}^{\mathrm{e}}$ et $\mathrm{XVII} \mathrm{I}^{\mathrm{e}}$ siècles est souvent identifiée au catholicisme, instrument idéologique au service de la monarchie confessionnelle des Habsbourg: les combats contre le crypto-judaïsme, contre l'érasmisme et le protestantisme, contre l'Islam jalonnent l'histoire de l'Espagne et du Portugal. Cette volonté de convertir a été exportée dans les territoires conquis puis colonisés et l'évangélisation des populations américaines a donné lieu à des controverses célèbres, à des récits nombreux de missions lointaines. Mais qu'en est-il des populations péninsulaires? N'y a-t-il eu qu'un catholicisme militant dirigé vers l'extérieur de la péninsule?

Les récits de missions intérieures témoignent pourtant du dynamisme de ce catholicisme tout aussi engagé que celui qui marque la politique internationale de l'Espagne et du Portugal de Philippe II. La réforme tridentine a mobilisé dans un effort important d'acculturation des masses les tribunaux inquisitoriaux, les évêques et les ordres religieux, notamment la Compagnie de Jésus qui a connu une expansion rapide dans la péninsule au cours de la deuxième moitié du xvi siècle. Si son implication dans la mise en place de la réforme tridentine est incontestable, la bibliographie reste cependant sommaire sur le déroulement de cette participation à l'éducation religieuse des populations ibériques ${ }^{1}$. L'existence de l'Inquisition, instrument de la Couronne, donne à l'histoire du catholicisme péninsulaire une nuance singulière grâce, en partie, à la capacité de l'institution à produire des archives. En effet, l'étude de la répression des minorités religieuses et des courants hétérodoxes dans la péninsule a retenu l'attention des chercheurs plus que la religion des « vieux chrétiens ». Ces derniers n'ont pourtant pas échappé au Saint-Office dans les moments de pénurie de groupes dissidents et d'offensives évangélisatrices de l'Église. La documentation inquisitoriale a servi à des analyses quantitatives et qualitatives sur la religion populaire mais presque toujours sous le prisme de la répression. Les archives jésuites permettent d'aborder le degré antérieur, en quelque sorte

1. Pablo Antón Solé, Los Pícaros de Conil y Zahara. Estudio histórico sobre los jesuitas y las almadrabas del duque de Medina Sidonia en la segunda mitad del siglo xV, Cadix, Jerez Industrial, 1965; Antonio Domínguez ORTzz, « Delitos y suplicios en la España imperial. La crónica negra de un misionero jesuita ", in ID., Crisis y decadencia de la España de los Austrias, Barcelone, Ariel, 1973, p. 13-71; Michele Ouvari, "Lettura "politica" dei resoconti missionari di Pedro de León, gesuita andaluso (1580-1620) ", Rivista di storia e letteratura religiosa, anno XII, 3, 1986, p. 475-491; Eugénio dos SANTos, "Missões do interior na região de Guimarães (séc. xviI) ", in Actas do Congresso histórico de Guimarães e sua Colegiada, Guimarâes, s. l., 1981, p. 219-236, et ID., " Missōes do interior em Portugal na época moderna. Agentes, métodos, resultados ", Arquipélago, 6, 1984, p. 29-65; Bernard VINCENT, « Jesuitas y Moriscos (1545-1570) », in ID., Minorías y marginados en la España del siglo xv, Grenade, Diputación provincial, 1987, p. 101-118. 
un seuil premier de l'« évangélisation ». Les initiatives de l'ordre fondé par Ignace de Loyola ont reçu l'appui tant du pouvoir central que des pouvoirs locaux grâce à l'intérêt politique et doctrinal qu'a soulevé la mise en place de la réforme de l'Église définie lors du concile de Trente. Il en a résulté une importante quantité de documents encore en friche. Leur analyse devrait permettre de prétendre à une meilleure connaissance des «Indes d'ici ", selon la terminologie même des missionnaires, des techniques employées et des finalités recherchées lors des missions.

Les récits des missions intérieures espagnoles ou portugaises à l'époque moderne ne sont pas différents de ceux d'autres régions d'Europe ${ }^{2}$. La correspondance entre les maisons de la Compagnie et Rome, lettres quadrimestres et annuae, les instructions des provinciaux ou du général, les histoires des collèges ou des provinces, les récits de missions ou les biographies de missionnaires constituent l'essentiel des fonds. La répétition d'un schéma épistolaire, l'apologie de l'action menée et l'absence systématique de toute forme d'échec, l'abondance de topiques pour décrire les lieux de missions, l'extraordinaire ou le merveilleux sont les notes dominantes de ces documents. Le travail des missionnaires est toujours décrit comme surhumain, exténuant; les missions, même longues de plusieurs mois, se déroulent à un rythme effréné car elles tentent de combler le vide religieux des campagnes péninsulaires. La prédication est présentée comme généreuse et didactique; en 1569 , pendant le Carême, un père et un frère jésuites parcourent le comté de Niebla, onze villages du sud de l'Espagne, où le profès prononce vingt-sept sermons car « dans neuf villages il n'y avait pas de prédicateur ${ }^{3} »$. La catéchèse est destinée aux enfants mais aussi aux villageois; au cours de la mission du comté de Niebla, le frère « enseigne la doctrine aux enfants et réunit les gens pour leur lire la vie des saints à l'aide d'un flos sanctorum ». Quant aux confessions, leur nombre

2. Voir, p. ex., Louis Châteller, La Religion des pauvres. Les sources du christianisme moderne, $X V^{2}-x I x^{2}$ siècles, Paris, Aubier, 1993; Bernard DoMPNIER, "La Compagnie de Jésus et la mission de l'intérieur ", in Les Jésuites à l'áge baroque, 1540-1640, éd. Luce Glard et Louis de Vaucelles, Grenoble, Jérôme Millon, 1996, p. 155-179; Carla Faralli, « Le missioni dei Gesuiti in Italia (sec. XVI-XVII). Problemi di una ricerca in corso », Bolletino della Società di studi Valdesi, anno XCVI, 138, 1975, p. 95-112; Luigi Fiorani, "Missioni della Compagnia di Gesù nell'agro romano nel xvu secolo ", Dimensioni e problemi della ricerca storica, 2, 1994, p. 216-234 ; David GENTILCORE, " "Accomodarsi alla capacità del popolo." Strategie, metodi e impatto delle missioni nel regno di Napoli, 1600-1800 ", Mélanges de l'École française de Rome (MEFRIM), t. CIX, 2, 1997, p. 689-722; Elisa Novi ChavarRIA "L'attività missionaria dei Gesuiti nel Mezzogiorno d'Italia tra XvI e xvil secolo », in Per la storia sociale e religiosa del Mezzogiorno d'Italia, éd. Giuseppe Galasso et Carla Russo, vol. II, Naples, Guida Editori, 1982, p. 159-185; Mario Rosa, « Strategia missionaria in Puglia agli inizi del Seicento ", in ID., Religione e società nel Mezzogiorno tra Cinque e Seicento, Bari, De Donato, 1976, p. 245-272.

3. Archivum Romanum Societatis Iesu (= ARSI), Hisp., 141, annua de 1568, Trigueros, $1^{\text {er }}$ janv. $1569, \mathrm{f}^{\mathrm{D}} 228 \mathrm{v}^{\circ}$. 
vertigineux est exprimé par les longues heures consacrées à ce ministère ou par une quantification hyperbolique : « Nous avons fait quelques missions importantes, un père a confessé en deux mois mille trois cents personnes et au cours de deux autres mois mille six cents ${ }^{4}$ ", informe le collège de Saint-Jacques de Compostelle en 1585. C'est d'ailleurs un passage obligé des récits de missions qui aboutit à la métaphore des moissons abondantes et, dans d'autres récits plus tardifs, aux exercices de pénitence. La relation se termine par les interventions des jésuites destinées à rétablir l'ordre moral par le bannissement de pratiques sociales ou culturelles jugées scandaleuses : dans le village de Fiñana, dans les Alpujarras, les couples vivent plusieurs années en concubinage avant de se marier, rapporte le jésuite en mission dans la région, en 1591; à Antequera, vers 1600, les prédicateurs «s'attachèrent à éradiquer un désordre scandaleux »: les femmes assistaient « librement» à des représentations théâtrales « où se commettaient beaucoup d'offenses à Notre Seigneur et c'était comme un séminaire de turpitudes et d'abominations ${ }^{5}$. Les médiations destinées à rétablir l'ordre politique viennent conclure les récits; en 1561, à Hallariz, en Galice, les missionnaires pacifient la communauté : «[...] sept morts d'hommes et d'autres maux ainsi que des scandales publics et de graves désordres ont été évités par la grâce du Seigneur grâce aux exhortations et confessions $^{6}$. » Mais c'est souvent un cas de conversion spectaculaire qui est rapporté à la fin des lettres, comme à Lucena, en 1620 : «La ville fut frappée par une conversion miraculeuse d'une personne qui scandalisait les habitants en raison de la vie dissolue qu'elle menait et après s'être convertie, elle tomba malade et mourut. Ceux qui la connaissaient en furent fort édifiés. » On retrouve les éléments de conversions semblables, souvent plus développés, dans beaucoup d'autres récits de missions : description de la faute (vie dissolue, des concubinages ou des adultères souvent) ou de la superstition (liée à un péché tu pendant de longues années), conversion grâce à la confession (les apparitions de la Vierge ou les tentations du démon sont fréquentes), maladie et mort du pécheur repenti, assuré du salut. Ainsi, si la structure du récit est fixe, le cas particulier introduit une variante qui est une sorte d'illustration finale du pouvoir des missionnaires à remettre de l'ordre dans le chaos initial, qu'il soit politique, moral ou religieux. Ces historiettes pourraient avoir dans le récit de mission, destiné à circuler, la même fonction que les exempla dans le sermon.

Cette forme du récit contribue à ritualiser l'action missionnaire et s'affirme surtout au XVII siècle lorsque la phase de reconquête des fidèles

4. Ibid.

5. Libro de la historia, principio, i progreso, del colegio de la Compañia de IHS de la ciudad de Antequera, Archivo histórico de la provincia de Toledo de la Compañía de Jesús (= AHPTCJ), C-195, $\mathrm{f}^{\mathrm{0}} 13 \mathrm{v}^{\circ}$.

6. ARSI, Hisp., 98 , Monterrey, 4 mai $1561, \mathrm{f}^{\text {os }} 183-184$. 
par l'Église, commencée, en Espagne et au Portugal, avant le concile de Trente, accélérée par celui-ci, est achevée et semble avoir porté ses fruits. En effet, dans le diocèse de Cuenca, les habitants traduits devant l'Inquisition, de tout âge et de toute condition sociale, ont réalisé d'énormes progrès dans l'apprentissage du catéchisme : si, dans la période 1544-1567, seulement un tiers des personnes interrogées connaissent les quatre prières de base, à la fin du siècle elles représentent $80 \%^{7}$. Même constat dans le district de l'Inquisition de Tolède : avant $1550,37 \%$ des accusés peuvent réciter sans erreur le Pater, l'Ave, le Credo et le Salve Regina, dans la période $1585-1599$, ils sont $68 \%$ et $82 \%$ entre 1600 et 1650 ; les chiffres sont semblables en ce qui concerne les commandements de Dieu ${ }^{8}$. Les missionnaires relayent d'ailleurs les institutions ecclésiastiques. La création, par exemple, de confréries du Doux Nom de Jésus instituées dans les villages pour éradiquer le blasphème fait suite à la campagne inquisitoriale de répression contre les «paroles scandaleuses » des années 1530-1560. On voit apparaître ensuite des confréries de dévotion du Rosaire qui perpétuent les enseignements des pères en mission qui distribuent des rosaires que les paysans portent sur eux comme des objets magiques. La constitution de ces associations pieuses correspond à un moment où les exigences envers les fidèles changent, au Xvil siècle, et les missions se transforment alors pour fixer, par une théâtralité accentuée, les acquis antérieurs.

Les missionnaires ont découvert, avec stupeur parfois, que les habitants des campagnes savaient à peine dire le Pater. En 1591, mais le thème est récurrent jusqu'au XviII ${ }^{e}$ siècle, le père Antonio de Torres dresse ce bilan :

«[...] considérez que presque tout le peuple chrétien n'a ni bases ni principes et que personne ne peut croire fidelater et firmenter [sic] ni espérer ni croire en Dieu ex charitate, personne ne connaît le culte qu'il faut rendre à Dieu ni aux choses sacrées, personne ne sait ce qu'est le Pater noster, l'Ave Maria et le Credo, personne ne sait se signer ni écouter la messe, personne ne connaît les obligations de son état car ce qui se passe généralement dans le christianisme, c'est qu'une fois l'enfant baptisé ni les parents ni les parrains ni les prélats ni les curés ne s'occupent de lui, où peut-il donc apprendre ces choses ${ }^{9}$ ?"

Andrés de Mora, en mission en 1592 dans la région de Guadix, durement éprouvée par la déportation des Morisques en Castille dans les années soixante-dix et repeuplée par des familles du nord de l'Espagne, décrit,

7. Voir Sara T. NALLE, God in La Mancha. Religious reform and the people of Cuenca, 1500-1650, Baltimore/Londres, Johns Hopkins University Press, 1992.

8. Jean-Pierre DEDIEU, « Catéchisme, communion, messe et confirmation dans l'archevêché de Tolède, 1540-1650 , Mélanges de la Casa de Velazquez, t. XV, 1979, p. 261-294.

9. Tomo $3^{\circ}$ de la Historia de esta provincia de Castilla de la Compañia de Jesús en que se continuan los varones ilustres de ella diuidido en otros cuatro libros, AHPTCJ, C-192, $\mathrm{f}^{\circ} 130$ (dans cet article, les traductions sont de M.-L. Copete et de F. Palomo). 
dans une lettre au général de la Compagnie, l'état d'ignorance des habitants de cette partie de l'Espagne mais aussi des prêtres car les bénéfices sont si minces qu'aucun ecclésiastique un tant soit peu lettré n'accepte de s'y rendre et les évêques « doivent y envoyer le premier venu aussi bête soit-il et c'est ainsi que dans presque tous les villages lorsque nous leur prêchions et enseignions les mystères de notre foi et le chemin du salut, ils découvraient toutes ces choses ${ }^{10}$ ". L'Espagne et le Portugal deviennent alors, dans les lettres envoyées à Rome, «Indes », «Indes du Japon » ou pays peuplés de « Maures blancs ». Pendant le règne de Philippe II, l'accent est donc mis avant tout sur l'évangélisation des populations rurales et l'instruction du bas clergé. Ainsi le déroulement de la mission est dicté par les besoins tout comme sa forme littéraire répétitive traduit une volonté d'homogénéisation de l'action; ce sont plutôt les faits exemplaires qui sont développés, les faits génériques étant fixes. Cette caractéristique se maintient encore longtemps, jusqu'à la fin des Temps modernes.

La mission du XVII ${ }^{e}$ siècle n'est cependant pas uniquement le résultat d'une transformation du contexte religieux, elle est tout autant le produit d'une expérience longue d'un demi-siècle au cours duquel la mission se définit par sa complexité. L'historien jésuite Mario Scaduto souligne en 1971 l'ampleur du contenu sémantique que le mot « mission » a acquis dans la correspondance de la Compagnie de Jésus au xvi siècle. En effet, par l'emploi de ce terme, on évoque, certes, l'activité développée auprès des infidèles, mais également des interventions apostoliques telles que les pèlerinages ou les pérégrinations des novices pour éprouver leur vocation, les voyages à caractère non religieux, les incursions dans les villages proches des collèges pour enseigner la doctrine chrétienne, les périples dans des pans entiers de diocèses à la demande des évêques, les campagnes de prédication faites pendant le Carême ${ }^{11}$.

Cette polysémie trouve sa justification dans la VII ${ }^{\mathrm{e}}$ partie des Constitutions de la Compagnie, où l'action apostolique des membres de l'Ordre est fondamentalement conçue comme une activité itinérante, marquée par la mobilité qui caractérisera l'identité jésuite ${ }^{12}$. Cette conception donne au terme « mission » un sens élargi comprenant des formes différentes d'apostolat qui, aujourd'hui, sont parfois considérées de manière fragmentaire, mais qui ont cependant des liens profonds entre elles et plus particulièrement en ce qui concerne leur dimension « politique ». En effet, la formation d'un homo civilis christianus est implicite dans toutes ces formes de

10. ARSI, Hisp., 134, Guadix, $1^{\text {er }}$ juillet 1592.

11. Mario SCADUTo, "La strada e i primi gesuiti », Archivum Historicum Societatis Iesu (= AHSI), t. XL, 1971, p. 323-390.

12. À ce propos, voir L. GiaRD, "Relire les Constitutions», in Les Jésuites à l'âge baroque, 1540-1640, op. cit. supra n. 2, p. 54-59. 
l'action apostolique jésuite, faisant de la mission un instrument de discipline sociale ${ }^{13}$. D'autre part, le lien entre ces différents types d'intervention, surtout dans le contexte européen, ne se définit pas seulement par leur conception, mais également par l'accomplissement de pratiques homogènes qui empêchent souvent une différenciation nette entre, par exemple, les pèlerinages et les campagnes de prédication ${ }^{14}$. Cependant, au cours de la deuxième moitié $\mathrm{du} \mathrm{xvI}^{\mathrm{e}}$ siècle, ce sont ces pratiques qui donnent son identité à la mission intérieure telle qu'on la pratique aux $\mathrm{XvII}^{\mathrm{e}}$ et XVIII ${ }^{e}$ siècles.

Le terme de «mission » va progressivement être utilisé dans la correspondance jésuite pour identifier un type spécifique d'apostolat que la Compagnie de Jésus développe dans les territoires de l'Europe catholique. Ainsi, dès les années 1570 , les litterae annuae portugaises et espagnoles mentionnent souvent des « missions » où l'on reconnaît une activité de prédication itinérante dont les origines se trouvent dans la tradition développée par les ordres mendiants au Moyen Âge. Cette tradition sera cependant renouvelée par les jésuites au xvi siècle ${ }^{15}$. Ces «missions", qui sont accomplies au moment du carême, de l'avent ou pendant les vacances scolaires, mènent, grâce à la détermination du général Claude Aquaviva, à l'institutionnalisation du ministère qui sera désigné par mission intra provinciam dans les années 1590 .

Cette activité apostolique acquiert un aspect pénitentiel qui caractérise au XvII siècle les missions intérieures ibériques ${ }^{16}$. En effet, malgré des allusions dans la correspondance à des missions accomplies en dehors du carême, l'action missionnaire des jésuites espagnols et portugais a, au

13. Sur le caractère «politique » des missions modernes, se reporter aux travaux d'Adriano ProsperI, « L'Europa cristiana e il mondo. Alle origini dell'idea di missione », Dimensioni e problemi della ricerca storica, 2, 1992, p. 189-220; et ID., Tribunali della coscienza. Inquisitori, confessori, missionari, Turin, Einaudi, 1996, p. 551-684.

14. Voir, p. ex., la description du pèlerinage accompli par Francisco Estrada à SaintJacques de Compostelle en 1546. Le père jésuite, sur injonction de ses supérieurs et avec le soutien probable de l'évêque, s'est arrêté deux mois à Porto où il va se consacrer à la prédication, à la visite des hôpitaux et des prisons, à la médiation et à la réforme des monastères; «Lettre de Francisco Henriques à Ignace de Loyola », Cö̈mbre, 23 nov. 1546, in Litterae quadrimestres, t. I, Madrid, Agustín Avrial, 1894, p. 17-27.

15. Sur les origines médiévales des missions jésuites, on trouve des notes relatives à l'activité développée par Vincent Ferrier et par Bernardin de Sienne, in L. Châtelujer, op. cit. supra n. 2, p. 17-21.

16. La caractérisation des missions intérieures (pénitentielles et doctrinales) a été établie par Giuseppe OrLaNDI, « Missioni parrocchiali e drammatica popolare », Spicilegium Historicum Congregationis SS.mi Redemptoris, t. XXII, 1974, p. 313-348. Il faut toutefois nuancer ce caractère pénitentiel des missions ibériques car l'activité doctrinale jouait aussi un rôle important. Dans la correspondance, on trouve des références à une activité quasi quotidienne de catéchèse pendant tout le temps de la mission mais aussi des mentions à l'organisation de petites représentations théâtrales à caractère doctrinal. Quelques-unes de ces pièces thêâtrales manuscrites sont conservées à la Biblioteca pública e Arquivo istrital de Évora (= BPADE), cód. CXIII/1-24, ${ }^{\text {os }} 172-216$. 
cours de la deuxième moitié du xvI siècle et de la première moitié du xvII ${ }^{e}$ siècle, un caractère fondamentalement quadragésimal. Le carême est conçu comme une période de préparation à Pâques; la confession et la communion sont alors obligatoires, et, par conséquent, c'est une période de réforme des individus, de réconciliation avec Dieu. Le contexte quadragésimal conditionne l'intervention des agents missionnaires et détermine les stratégies des missions réalisées en dehors de ce temps liturgique. Ces missions sont, d'ailleurs, considérées comme des "carêmes" après le Carême ${ }^{17}$. Par exemple, le recours aux thématiques centrées sur la passion du Christ et l'accomplissement de disciplines publiques constituent des éléments propres de ces célébrations. Ces aspects s'intègrent en même temps dans la procédure des missions intérieures qui acquièrent ainsi une teneur pénitentielle vouée à la "conversion » des fidèles. Cet objectif exige du missionnaire la mise en scène de certaines stratégies de communication dont les caractéristiques se dessinent à travers l'activité apostolique et culminent dans la deuxième moitié du xviı siècle.

En 1624, João Pereira, dans une lettre au recteur du collège d'Évora, raconte les événements de la mission d'Alcácer do Sal. Parmi les différentes médiations des deux missionnaires dans des litiges, Pereira énumère les efforts, que lui et son compagnon ont déployés pour obtenir le pardon de la famille d'un homme assassiné. Convaincus de l'innocence de l'accusé emprisonné et après une première tentative infructueuse, les deux religieux décident de se rendre chez la veuve. Ils s'agenouillent et commencent à pleurer devant le crucifix « très dévot » qu'ils ont apporté. Devant l'obstination des offensés, l'un des pères jésuites leur rappelle l'obligation chrétienne du pardon aux ennemis, à l'instar du Christ. Finalement, émus par les propos du religieux, la femme et le jeune homme embrassent le crucifix et, en pleurant, accordent le pardon ${ }^{18}$.

Toute la description de la scène est marquée par une dramatisation qui caractérise souvent la rhétorique des lettres missionnaires, mais qui, en même temps, reflète la réalité des stratégies mises en place pendant la mission afin de «mouvoir » les fidèles à la «conversion». Le succès de la mission est mesuré, selon des critères constants, en partie à l'aune des conflits résolus et des confessions réalisées. Toutefois, on trouve aussi des manifestations bouleversantes dans lesquelles les cris et les larmes des

17. Ainsi, le P. Frutuoso ANDrÉ, envoyé en 1552 en mission quadragésimale à Lagos, au sud du Portugal, se référait aux campagnes de prédication qu'il avait, par la suite, accomplies dans toute la région, en disant qu' "après le Carême, un autre carême avait commencé », «Lettre de Frutuoso André à Ignace de Loyola », Algarve, 22 juil. 1552, in Litterae quadrimestres, t. I, op. cit. supra n. 14, p. 716.

18. João Pereira, Carta da missam da Villa de Alcacere do Sal, para o padre Francisco de Mendoça reitor do collegio da Companhia de Jesv e Vniuersidade de Euora (1624), Biblioteca nacional de Lisboa (= BNL), ms. 30, $\mathrm{n}^{\circ} 213, \mathrm{f}^{\circ} 2 \mathrm{v}^{\circ}$. 
fidèles constituent les signes extérieurs d'une réconciliation soudaine avec Dieu. La représentation de ce genre de conversion, à travers l'image de l'homme qui pleure, est attestée dans la correspondance des jésuites mais aussi dans l'iconographie de l'époque ${ }^{19}$, et renvoie à la théorie des mouvements de l'âme et de leur extériorisation de la tradition aristotélicienne du De anima $(\mathrm{I}, 1)$. Ces fondements culturels façonnent le regard du missionnaire et peuvent conditionner en même temps les stratégies de communication dont il se sert au cours de la mission. En effet, les stratégies visant à toucher psychologiquement les fidèles, telles celles décrites par Pereira, mettent en relief l'élaboration des méthodes missionnaires en fonction des réponses que le religieux espère obtenir de la part des fidèles. Ainsi, à travers les différentes stratégies utilisées, des jeux d'interaction s'établissent entre la population et le jésuite même; les formes de « présentation » constituent le premier des aspects qui conditionnent le succès de son intervention auprès de la communauté ${ }^{20}$. Que ces formes de présentation soient décidées au début de la mission ou que les religieux les élaborent au cours de celle-ci, elles répondent au besoin d'«acquérir de l'autorité et de gagner la confiance » des fidèles.

Ces objectifs sont clairement établis dans les instructions relatives à l'accomplissement des ministères de la Compagnie élaborées au Xvi ${ }^{\mathrm{e}}$ siècle. L'importance que certaines de ces règles donnent aux entrées des missionnaires traduit bien l'intérêt du premier contact. En 1610, les avis rendus à ce sujet par le visiteur de la province portugaise, le père João Álvares, illustrent grosso modo la méthode à suivre. Les deux opérations fondamentales se définissent par la présentation aux autorités de la ville et l'annonce de la mission lors du premier sermon ${ }^{21}$. La méthode du père Álvares correspond, en réalité, à celle que l'on trouve décrite, de manière plus ou moins précise, dans la correspondance des provinces ibériques ou d'autres régions de l'Europe catholique ${ }^{22}$. Toutefois, l'arrivée des missionnaires dans la ville a souvent pris des traits cérémoniels particuliers, surtout lorsqu'il s'agit d'une mission de carême. En effet, on trouve dans les

19. P. ex., la conversion était représentée dans l'Iconologia de Cesare RiPA, Rome, 1593, par une femme pleurant qui avait à ses pieds des vêtements luxueux, des bijoux et les cheveux coupés. À propos de ce genre de manifestations extemes de la conversion, voir Esther CoHEN, «Fire and tears. The physical manifestations of conversion », Dimensioni e problemi della ricerca storica, 2, 1996, p. 247-253.

20. Sur le rôle des formes de «présentation » dans les jeux d'interaction, voir le travail classique d'Erving Gorfman, The Presentation of self in everyday life, Garden City, NY, Doubleday, 1959.

21. Visita da prouincia de Portugal, pelo P.e Joāo Aluares, Arquivo nacional da Torre do Tombo (= ANTT), Armário Jesuítico, liv. V, [1610], p. 46.

22. C. Faralli, art. cit. supra n 2, p. 108; et Maria Gabriella Rienzo, « Il processo di cristianizzazione e le missioni popolari nel Mezzogionno. Aspetti istituzionali e socio-religiosi ", in Per la storia sociale e religiosa del Mezzogiorno d'Italia, op. cit. supra n. 2, vol. 1, 1980, p. 450 . 
documents de multiples références à l'accueil des pères jésuites par les autorités locales avant même qu'ils aient franchi les portes de la ville. Ensuite, un cortège est organisé et parcourt les rues de la localité. Enfin, d'autres références évoquent la visite de notables aux religieux ${ }^{23}$. Ces initiatives, bien que modestes, ressemblent aux rituels d'entrée réservés, à la même époque, à certaines autorités civiles et ecclésiastiques et, notamment, à des visiteurs inquisitoriaux ou épiscopaux ${ }^{24}$.

$\mathrm{Si}$ nous considérons ces pratiques cérémonielles comme des « rites d'institution ${ }^{25}$ », les entrées des missionnaires évoquées ci-dessus peuvent être comprises comme des stratégies de communication. En effet, la qualité de l'accueil réservé aux missionnaires permet de mettre en exergue la distinction qui s'opère entre les missionnaires, considérés comme des agents extraordinaires, spécialisés dans les ministères de la prédication et de la confession, et les membres du clergé local. À la lumière de ces cérémonies, on reconnait l'autorité dont jouissent les missionnaires jésuites, autorité qui leur octroie non seulement le droit de pardonner les péchés mais aussi d'intervenir dans la vie de la communauté ${ }^{26}$.

Cette supériorité du missionnaire jésuite exige de lui l'accomplissement d'autres stratégies plus subtiles mais peut-être plus efficaces, dans la mesure où son comportement doit répondre aux attentes de l'ensemble de la population qui lui attribue son statut. La construction d'une image du jésuite devient ainsi l'un des traits de la méthode missionnaire qui est évoquée systématiquement dans les rapports de mission. Cette représentation traduit bien des formes d'« autoperception » des jésuites dans le cadre de leur apostolat. Par exemple, Diogo Ferreira décrit ainsi la réaction des habitants de l'évêché de Guarda vis-à-vis de son comportement et de celui du père Sebastião Barradas, lors d'une mission de carême en 1594 :

23. Voir, p. ex., la description de l'entrée en 1570 des missionnaires dans la ville de Crato, siège d'un des ordres militaires portugais, où ils ont été accueillis aux portes de la ville par le maîttre prieur Dom ANTónıo, fils illégitime du prince Dom Luís : Carta que o P.e Garcia Simões escreueo do Crato aonde foi em Missão em companhia do P.e Miguel de Sousa a 18 de Feuereiro de 1570, in Segundo tomo das cartas de Europa. Do anno de 1560 ate o anno de 1575 , BPADE, cód. CVIII/2-2, fos $305 \mathrm{v}^{\circ}-307 \mathrm{v}^{\circ}$. Des cérémonies similaires sont encore rapportées dans les lettres de missions de Coruche (1624) et de Setúbal (1650) : voir Sebastião Alvares, Carta da missam da villa de Curuche para o P.e Francisco de Mendoça da Companhia de Jesus, reitor do collegio e vniuersidade de Euora, BNL, ms. $30, \mathrm{n}^{\circ} 215$; et Bartolomeu de BRrTo, Carta da missão de Setuval, pera o P.e Francisco Cabral, da Companhia de Jesus, reitor do collegio, e vniuersidade do spirito santo, em Euora. De Setuval, 20 de Abril de 1650, BNL, ms. $30, \mathrm{n}^{\circ} 214$.

24. À propos des cérémonies d'accueil, dans le monde ibérique, des visiteurs inquisitoriaux et épiscopaux, voir Francisco Bethencourt, L'Inquisition à l'époque moderne. Espagne, Portugal, Italie, $X V^{\mathcal{E}}$-XIX $X^{\varepsilon}$ siècles, Paris, Fayard, 1995, p. 229-232.

25. Pierre Bourdieu, "Les rites comme des actes d'institution ", Actes de la recherche en sciences sociales, 43,1982 , p. 58-61.

26. Les missionnaires jouissaient généralement du privilège d'absolution des cas réservés. Ces privilèges étaient accordés par les évêques et, à partir du xvi" siècle, par le pape. 
« Je ne puis trouver les mots pour parler à Votre Révérence du crédit que dans cette région on accorde à la Compagnie; les gens nous obéissent comme si nous étions des hommes envoyés par le Ciel; dans les rues, ces gens ne disent qu'une chose : que Dieu vient les visiter à travers ces saints hommes et que si ces derniers étaient venus chaque année dans ces parages il n'y aurait pas eu autant de péchés. Ces gens sont très édifiés de nous voir nous déplacer avec nos bourdons et nos besaces à la recherche d'hôpitaux et de greniers où passer la nuit même si les notables de cette région le déplorent ${ }^{27}$."

Leur attitude encourage les fidèles à l'obéissance. Ces comportements favorisent probablement leur disposition envers les missionnaires qui laissent apparaître des signes extérieurs de pauvreté religieuse tels que le fait de marcher à pied, l'usage du bourdon de pèlerin et de la besace utilisée généralement par les prédicateurs des ordres mendiants.

Par ailleurs, depuis sa fondation, la Compagnie de Jésus a accordé une attention particulière à la conduite de ses membres au cours de leur action apostolique. Les Règles de la modestie, élaborées en 1555, montrent bien l'intérêt des supérieurs de l'ordre ignatien pour cette question. Ces sont des instructions précises sur la manière dont le jésuite doit contrôler son expression, ses gestes et ses mouvements et comment il doit s'adresser à son prochain ${ }^{28}$. Il s'agit, en effet, d'une discipline du corps à travers laquelle la disposition intérieure du sujet est extériorisée. L'origine de ces règles se trouve dans la tradition disciplinaire monastique et, notamment, dans l'œuvre de Hugues de Saint-Victor, De novitiorum institutione, qui sera d'ailleurs largement diffusée en dehors des milieux religieux. En effet, la modestie, telle qu'elle a été conçue par Hugues de Saint-Victor, est à l'origine de toute une littérature normative séculière qui investit cette notion d'une fonction "politique » et qui la transforme, au XVI siècle, en l'un des paradigmes du bon comportement ${ }^{29}$, voire de la "discipline

27. Missions du collège de Cö̈mbre (Carême, 1594), ARSI, Lus., 106, Historia, fo $183 \mathrm{v}^{\circ}$.

28. Monumenta Ignatiana. Series tertia, t. IV : Regulae Societatis Iesu (1540-1556), Rome, IHSI, 1948, p. 518-526.

29. Les travaux sur la littérature des «bonnes manières » et leur caractère politique sont abondants, notamment depuis l'attention portée par l'historiographie à l'cuuve de Norbert Elias sur le «procès de civilisation ». Toutefois, à propos de la tradition monastique, qui était à la base de cette littérature, voir Dylwin KNOX, " "Disciplina". The monastic and clerical origins of the European civility ", in Renaissance society and culture. Essays in honour of Eugene F. Rice, Jr., éd. John Monfasani et Ronald G. Musso, New York, Italica Press, 1991, p. 107135; ID., « Disciplina. Le origini monastiche e clericali del buon comportamento nell'Europa cattolica del Cinquecento e del primo Seicento ", in Disciplina dell'anima, disciplina del corpo e disciplina della società tra medioevo ed età moderna, éd. Paolo Prodı, Bologne, Il Mulino, 1994, p. 63-99; Daniela Romagnol, " La courtoisie dans la ville. Un modèle complexe ", in La Ville et la cour. Des bonnes et mauvaises manières, éd. D. Romagnou, Paris, Fayard, 1995, p. 25-87; ID., " "Disciplina est conversatio bona et honesta" ". Anima, corpo e società tra Ugo de San Vittore ed Erasmo de Rotterdam ", in Disciplina dell'anima, disciplina del corpo e disciplina della società tra medioevo ed età moderna, op. cit. supra, 
sociale ${ }^{30}$. Bien que les Règles de la modestie soient directement rattachées à la tradition monastique, la vocation jésuite de "vivre dans le siècle » donne à ces règles une autre dimension et permet d'ailleurs d'établir des liens entre les textes normatifs visant le comportement des membres de l'Ordre et la littérature des «bonnes manières » de cette époque. Ces liens sont manifestes dans les Industrias de Juan de Polanco, qui s'avèrent intéressantes, non seulement par leur contenu, mais également en raison de leur diffusion dans la péninsule ibérique ${ }^{31}$. Le texte, qui constitue une sorte de brouillon et de commentaire aux Constitutions de la Compagnie de Jésus, réunit sous le sixième titre les codes de comportement jésuites, auxquels Polanco ajoute des recommandations pour chaque ministère, celui de la «conversation» en particulier. Ils s'articulent autour de quatre moyens fondamentaux qui permettent de mener à bien la vocation d'aide au prochain des compagnons d'Ignace de Loyola. La vie exemplaire du religieux, la manière de gagner la confiance des fidèles, la crédibilité et l'autorité, la connaissance du milieu où le jésuite est appelé à intervenir charpentent le texte des Industrias. Les stratégies présentées par Polanco pour offrir une image « vertueuse » du jésuite s'organisent autour de l'aspect extérieur, la retenue des mots et des gestes, le mépris pour les choses temporelles, l'humilité, le refus des ostentations doctrinales, la patience et la prudence. En effet, cette figure apparaît en filigrane dans les Industrias comme un processus de communication mettant en scène des formes de présentation

p. 507-537; Giovanni Pozzı, « Occhi bassi », Thematologie des Kleinen. Petits thèmes littéraires, éd. Edgar Marscho et Giovanni Pozzı, Fribourg, Éditions Universitaires, 1986, p. 185-191.

30. Sur le concept de "discipline sociale " et le rôle joué par celle-ci dans l'histoire politique de l'Europe occidentale, voir Gerhard Oestreich, "The structure of the absolute state ", in ID., Neostoicism and the early modern state, Cambridge, Cambridge University Press, 1982, p. 258-273; Winfried ScHuLze, « Il concetto di “ disciplinamento sociale nella prima età moderna " in Gerhard Oestreich ", Annali dell'Istituto storico italo-germanico in Trento, vol. XVIII, 1992, p. 371-411; Pierangelo SCHIERA, «Disciplina, disciplinamento », Annali dell'Istituto storico italo-germanico in Trento, vol. XVIII, 1992, p. 315-334; ID., " Disciplina, Stato moderno, disciplinamento. Considerazioni a cavallo fra la sociologia del potere e la storia costituzionale », in Disciplina dell'anima, disciplina del corpo e disciplina della società tra medioevo ed età moderna, op. cit. supra n. 29, p. 21-46; P. Prodl, "Presentazione », in ibid., p. 9-17.

31. Juan DE Polanco, Industrias con que uno de la Compañía de Iesús mejor conseguirá sus fines, in Polanci complementa. Epistolae et complementa P. Joannis Alphonsi de Polanco Societate lesu, t. II, Madrid, Gabriel López del Horno, 1917, p. 776-807. Une copie manuscrite de ce texte se trouve dans les instructions données par Jérôme Nadal aux jésuites portugais en 1561 (ARSI, Institutum, 208), d'autres copies sont conservées dans des volumes portugais qui contiennent des règles de la Compagnie et qui appartenaient probablement à des novices ou à des maîtres de novices jésuites (BNL, cód. 3648 et cód. 4214). Pedro de León incluera le texte dans les volumes relatifs aux missions qu'il avait faites dans la deuxième moitié du xvi ${ }^{e}$ siècle et au début du xviI siècle, voir Pedro de León, Compendio de algunas experiencias en los ministerios de que usa la Compañía de Jesús, con que prácticamente se muestra con algunos acontecimientos y documentos el buen acierto en ellos por órden de los superiores por el padre Pedro de León de la misma Compañía. Año de 1619, Biblioteca universitaria de Granada (= BUG), caja B-76. 
conçues pour avoir un impact efficace sur les fidèles. Toutefois, l'extériorisation de telles vertus ne peut pas être considérée uniquement comme le résultat d'une action préméditée, mais également comme le produit d'un habitus propre au jésuite, intériorisé à l'issue d'une éducation spirituelle essentiellement vouée à l'intervention apostolique.

La « présentation » des jésuites devient ainsi une stratégie essentielle des formes d'interaction entre les missionnaires et les communautés de fidèles. Elle dispose favorablement les populations visitées envers les pères missionnaires et elle prépare les objectifs finaux de conversion recherchés à travers la mission. Toutefois, cette finalité mène à la mise en scène d'autres tactiques dont la charge dramatique a des conséquences visibles sur l'esprit des fidèles poussés au repentir, à la confession et à la réforme des mœurs. Il peut s'agir de visites d'hôpitaux, d'assistance aux pauvres ou de pacifications. Elles trouvent cependant leur cadre le plus adapté dans la prédication, où le missionnaire se sert de son habileté rhétorique, mais aussi d'autres moyens non discursifs qui frappent le public.

En effet, la prédication constitue, avec la confession et la catéchèse, le ministère le plus important de la mission. Il s'agit cependant d'un genre de prédication qui va progressivement se distinguer des autres formes de l'éloquence sacrée des $X \mathrm{XI}^{\mathrm{e}}$ et $\mathrm{XVII}{ }^{\mathrm{e}}$ siècles, surtout des sermons à caractère cérémoniel prononcés généralement à l'occasion de fêtes ou d'événements importants. Toutefois, le nombre d'études qui ont abordé la prédication missionnaire à l'époque moderne est encore réduit et les sources sont parfois peu prolixes en raison même de la nature d'un genre qui ne suppose pas toujours un travail d'écriture. Il est cependant possible de saisir les caractéristiques de la prédication missionnaire depuis la fin du xvi siècle, tant en ce qui concerne la rhétorique utilisée, le langage employé et les thématiques abordées que la mise en scène des sermons, à travers l'étude de quelques collections de sermons imprimés et manuscrits ${ }^{32}$, ou, encore, de descriptions d'interventions de prédicateurs rapportées dans la correspondance.

Pedro de León ${ }^{33}$ n'hésite pas à écrire qu'il n'a pas « le talent des prédicateurs qui occupent des chaires importantes en ville " mais que les pères plus aptes à la prédication missionnaire sont « ceux qui ne sont pas célèbres

32. La première compilation imprimée de sermons de mission ne date que de 1652 . Il s'agit de la Predicación fructuosa du P. Pedro Jerónimo Contınente publiée à Saragosse par Diego Dormer. Autres collections de sermons manuscrits d'auteurs ayant développé une activité apostolique : Inácio Martins, Sermões, BNL, cód. 3501-3503 et 6271; Gaspar FerNANDES, Sermōes, BNL, cód. 3021.

33. Le jésuite andalou Pedro de LEón (1545-1632) est surtout connu pour avoir exercé, à Séville, le ministère des prisons mais parallèlement à cette activité, il a effectué de nombreuses missions dans toute l'Andalousie et en Estrémadure. Dans son Compendio, op. cit. supra n. 31, il fait le récit de sa carrière au sein de la Compagnie de Jésus. 
car il n'y a pas de mauvais pain si la famine est grande ${ }^{34}$. Les transformations que le Portugais Inácio Martins a introduites dans sa prédication dans les années 1570 suivent des directions similaires aux indications données par Pedro de León. Le brouillon d'une biographie anonyme sur ce père jésuite souligne d'abord sa vie exemplaire. Il a quitté les chaires de la cour royale où il a prêché longtemps « avec un style beaucoup plus concerté et apprêté ». D'une façon impressionniste, le texte met en relief les différences qui séparent une prédication solennelle, celle qu'exigeait un contexte courtisan, de la prédication apostolique, identifiée à l'activité que, dans ce domaine, Martins a développée pendant les vingt dernières années de sa vie.

Le texte nous permet de déceler les contours d'une prédication qui n'est pas destinée à délecter son public ou seulement vouée à réformer les mœurs mais qui exige aussi un grand talent de communication. La performance ou, si l'on préfère, l'actio et l'emploi d'un style simple se placent au centre de la stratégie du prédicateur à côté de sa capacité à conduire les esprits des fidèles à la « conversion » en suscitant la crainte de leurs péchés par le recours à des formes rhétoriques ou par l'emploi des « espèces sensibles $^{35} »$. Celles-ci, d'ailleurs, deviennent parfois l'un des éléments spécialement détaillés dans les références aux prédications qui sont rapportées dans les relations des missions et dans les litterae annuae. En 1571, par exemple, pendant le sermon prononcé le dimanche de la saint Lazare dans un village des alentours d'Évora, le prédicateur a soudain montré un crâne avec lequel il s'est entretenu, matérialisant ainsi aux yeux des fidèles l'image de la mort qui était probablement au centre de la thématique abordée dans la prédication. Le coup de théâtre du jésuite, accompli à la fin du sermon, « le prédicateur et l'auditoire étant fort émus ", reçoit une réponse immédiate de la part des fidèles qui commencent à crier et à pleurer, enlèvent leurs bijoux, décident de réformer leurs modes de vie et d'abandonner leur « mauvais état ${ }^{36}$.

Alors qu'ils prêchent, les missionnaires font souvent appel à des images de la passion du Christ ou à des crucifix dont la fonction est d'impressionner le public ${ }^{37}$. Il faut cependant souligner la relation étroite qui généralement existe entre l'emploi de ces « espèces sensibles », assez répandues

34. P. DE LEón, Compendio de algunas experiencias en los ministerios de que usa la Compañia de Jesús, con que prácticamente se muestra con algunos acontecimientos y documentos el buen acierto de ellos, repr. in Pedro Herrera Puga, Grandeza y miseria en Andalucía. Testimonio de una encrucijada histórica, Grenade, Facultad de Teología, 1981, p. 167.

35. Da uida e uirtudes do Santo Varão Mestre Ignacio Martins, BPADE, cód. CIX/2-13, peça $7, f^{u s} 84 v^{\circ}-85$. $\mathrm{v}^{\mathrm{0}}$.

36. Luis Perpiñ́n, Annua de la province portugaise de 1571, ANTT, Livraria, ms. 690, fo 5

37. Pour d'autres exemples, Fernáo GuerreIro, Annua da prouincia de 80, ANTT, Livraria, ms. $690, \mathrm{f}^{\mathrm{v}} 74 \mathrm{v}^{\circ}$, et J. Pereira, doc. cit. supra $\mathrm{n} .18, \mathrm{f}^{\mathrm{o}} 1 \mathrm{v}^{0}$. 
dans la prédication du XVII ${ }^{\mathrm{e}}$ siècle $^{38}$, et le texte du sermon. En effet, celui-ci contribue aussi à la dramatisation de la prédication à travers, par exemple, l'introduction de formes dialoguées, la création d'images mentales grâce à des descriptions. C'est le cas du sermon du chemin de croix prononcé par le père Gaspar Fernandes dans la ville d'Arraiolos en $1618^{39}$. Le jésuite développe progressivement le versant tragique des scènes qu'il décrit, renforcé par les images successives du Christ qui porte la croix pour rédimer l'humanité. La première de ces images le montre lors de sa première chute :

«Le Seigneur marchait vers le Calvaire par une rue qui descendait et, quand il arriva à la fin de cette rue, après avoir fait quatre-vingts pas, il commença à défaillir. Il était couvert de sueur froide, il était livide, il blêmissait, il était épuisé et hors d'haleine et, ne pouvant plus supporter le poids de la croix, il s'évanouit ${ }^{40}$."

Ensuite, la description de la rencontre de Jésus-Christ avec sa mère prépare émotionnellement l'auditoire aux scènes que le prédicateur va continuer à évoquer pendant le sermon. L'aide de Simon de Cyrène nous présente une nouvelle image du Christ dans laquelle Fernandes a recours à des éléments du langage qui relèvent du pathétique :

"Son visage où se lisait l'épuisement était couvert de sang et de sueur, meurtri. Il était blessé à la tête, il avait les yeux mi-clos et les cheveux en désordre tant on les lui avait tirés; bref, il était dans un tel état qu'il pouvait mouvoir les cours les plus durs à la douleur et à la pitié ${ }^{41}$. "

Le sermon s'achève par un dernier épisode montrant sainte Véronique qui essuie le sang du Christ, entourée d'autres femmes qui pleurent le sort du Seigneur. La scène vise probablement à provoquer dans l'auditoire des effets similaires à ceux relatés par le prédicateur ${ }^{42}$. Le recours aux descriptions dans la prédication est une forme rhétorique bien connue, qui sera de plus en plus utilisée, et qui rappelle d'ailleurs les techniques de composition de lieu caractéristiques des exercices spirituels d'Ignace de Loyola. Si l'objectif de ces images mentales est celui d'émouvoir l'auditoire qui assiste au sermon, l'effet peut être encore amplifié par l'emploi d'images

38. Pour le contexte ibérique, voir Giuseppina LEDDA, « Forme e modi di teatralitá nell'oratoria sacra del Seicento », Studi ispanici, 1982, p. 87-107; et ID., “ Predicar a los ojos », Edad de Oro, 8, 1989, p. 129-142.

39. G. Fernandes, « Sermão da procissão dos passos " (Arraiolos, 1618), in op. cit. supra n. 32 , vol. II, $\mathrm{f}^{\text {s }} 143-144 \mathrm{v}^{\circ}$.

40. Ibid., $\mathrm{f}^{\circ} 143 \mathrm{v}^{\circ}$.

41. Ibid, $\mathrm{f}^{\circ} 144$.

42. Ibid. 
réelles qui renforcent ainsi celles créées par le discours. Les réponses des fidèles à l'intervention du prédicateur mettent encore en exergue les jeux d'interaction qui s'établissent entre la population et le missionnaire. Celui-ci doit recourir à ces stratégies discursives et non discursives afin de provoquer dans l'auditoire des réactions d'un fort contenu dramatique (pleurs, flagellations, pardons publics) ${ }^{43}$ auxquelles contribue aussi l'image du missionnaire lorsqu'il prêche. Louis de Grenade, auteur d'un des manuels d'éloquence sacrée les plus diffusés dans la péninsule ibérique, affirme que la " prononciation » dans le sermon « engendre dans les âmes du public des mouvements et des émotions provoqués par la voix, le visage et les gestes de celui qui parle ${ }^{44} \gg$. Le message transmis par la prédication trouve ainsi l'un de ses supports essentiels dans la figure du prédicateur qui devient une sorte de miroir qui convie les fidèles à imiter les sentiments exprimés par le missionnaire au long du sermon. En outre, l'image du prédicateur va devenir de plus en plus complexe. L'emploi de disciplines, de la corde autour du cou et de la couronne d'épines, deviennent une pratique assez fréquente dans la prédication missionnaire de la deuxième moitié du XvII ${ }^{e}$ siècle. Le prédicateur se montre ainsi devant l'auditoire comme l'alter Christus, qui rachète les péchés des fidèles et qui les exhorte, par l'imitation, à la pénitence ${ }^{45}$.

L'origine de ces stratégies se trouve dans les relations qui sont établies entre le texte du sermon et l'actio, c'est-à-dire la voix, le ton, la prononciation et les gestes du prédicateur. Cette partie de l'ars rhetorice a été revalorisée à l'époque grâce aux efforts de rénovation que la prédication a connus depuis le concile de Trente. En effet, les instructions épiscopales et les nombreux traités d'éloquence sacrée publiés après la clôture du Concile, donnent un poids nouveau à l'actio. Ces manuels soulignent d'une façon explicite la concordance qui doit exister entre ce que le prédicateur dit dans le sermon et la façon dont il le formule devant un auditoire ${ }^{46}$. L'accentuation de l'actio dans la prédication missionnaire contribuait à la « dramatisation » de ce ministère. Celle-ci n'est pas étrangère aux jésuites dont l'enseignement a trouvé dans le théâtre scolaire un des exercices essentiels de

43. Ces réactions sont largement rapportées dans les récits de mission. Voir, p. ex., Luís DA CRUz, Annua de 1579, ANTT, Livraria, ms. 690, $\mathrm{f}^{\mathrm{0}} 66 \mathrm{v}^{\circ}$.

44. Louis DE GRENADE, O.P., Los seis libros de la retórica eclesiástica o de la manera de predicar, 1576, in ID., Obras, Madrid, M. Rivadeneyra, 1879, p. 614.

45. Bernadette Majorana, Aspetti performativi e spettacolari delle missioni popolari. L'esperienza gesuitica nell'Italia centrale tra Sei e Settecento, thèse de doctorat, Florence, università degli Studi di Firenze, 1995; et ID., " Elementi drammatici della predicazione missionaria. Osservazioni su un caso gesuitico tra xvir e xvil secolo ", in La Predicazione in Italia dopo il concilio di Trento tra Cinquecente e Settecento. Atti del X Convegno dell'Associazione Italiana dei professori di Storia della Chiesa. Napoli, 6-9 settembre 1994, éd. Giacomo Martina et Ugo Dovere, Rome, Dehoniane, 1996, p. 127-152.

46. Voir, p. ex., L. DE GRENADE, op. cit. supra n. 44, p. 613-630. 
l'apprentissage rhétorique. Les rapports entre prédication et théâtre ont été fréquemment étudiés. Les parallélismes entre ces deux activités, qui sont plus forts dans le thêâtre religieux, se réfèrent aux auditoires, aux espaces (par exemple, le temple baroque comme espace théâtralisé), à l'énonciation, aux structures dramatiques des textes, et aux appareils non discursifs utilisés ${ }^{47}$. Il est toujours possible de trouver des racines communes quant aux processus de formation des agents engagés dans une activité et dans l'autre. Toutefois, des travaux récents ont souligné le besoin de nuancer ces liens dans le cas de la prédication missionnaire qui, pour ce qui est des styles propres de la mise en scène théâtrale et du spectacle baroque, présente des traits spécifiques. Il s'agit d'une méthode qui utilise des éléments propres à la culture urbaine mais qui les adapte aux exigences d'un contexte rural dans lequel la mission se développe. Ainsi, les systèmes de communication sont simplifiés, non pas au niveau de la diversité des formes employées, mais au niveau des acteurs et des instruments utilisés. Le missionnaire, en effet, se place au centre d'une performance qui doit mener l'ensemble de la population à une participation active dans les divers actes qu'il accomplit; cette participation est inconnue dans la prédication solennelle, plus directement liée aux styles théâtraux ${ }^{48}$. Cette méthode prend des traits plus marqués lors de la prédication; elle est également présente dans d'autres interventions du missionnaire qui essaye ainsi de mener les fidèles à l'imitation et à l'objectif final qu'est la conversion. Celle-ci, en accord avec l'idée de la mission moderne, acquiert également un sens «politique », qui trouvera dans l'action de pacification sa manifestation la plus remarquable.

La mission n'a pas à l'époque moderne qu'une fonction religieuse. Les missionnaires jésuites au cours de leurs périples remplissent aussi le tôle de pacificateurs des conflits sociaux ou politiques dans les villages qu'ils traversent. C'est un rôle traditionnel dévolu aux membres du clergé : on les voit jouer les intermédiaires entre débiteurs et créanciers dans les procès civils, entre accusés et victimes pour les pardons de la partie offensée dans les causes criminelles. Mais il s'agit ici d'un autre niveau de médiation, désigné dans les récits de missions sous le terme de pacifications (paces) ou d'amitiés (amistades). Il s'agit de conflits internes à la communauté qui se cristallisent en haines, dégénèrent en violences entre familles rivales ou partis opposés, car tout habitant est tenu de choisir son camp. C'est souvent

47. P. ex., Emilio Díaz Orozco, « Sobre la teatralización del templo y la función religiosa del barroco. El predicador y el comediante (notas a una "Introducción al barroco") », Cuadernos para la investigación de la literatura hispánica, 2-3, 1980, p. 171-188; G. LEDDA, art. cit. supra n. 38, 1982, passim.

48. B. Majorana, Aspetti performativi e spettacolari delle missioni popolari, thèse citée supra ก. 45 , passim. 
le fait du patriciat qui lutte pour s'approprier le pouvoir municipal. Les habitants sont ainsi pris en otages. La médiation des missionnaires n'a alors d'autre but que de réduire les différences, de gommer les forces politiques qui se singularisent pour restaurer la paix troublée.

Un des exemples les plus intéressants, parce qu'il est extrême et permet de démonter les mécanismes de la médiation, est celui des madragues dans la deuxième moitié du xvi siècle. L'une des missions traditionnelles des jésuites du collège de Séville depuis 1557 est celle de la côte gaditane, entre Tarifa et Cadix, dans les pêcheries de thon du duc de Medina Sidonia. Elle a lieu tous les ans depuis 1557 et elle est attestée jusqu'au début du xvir siècle. Elle éveille les vocations missionnaires. Après la mission des pères Diego Lopez et Gregorio Mata, en 1558, les candidatures abondent. Dans son histoire de la province jésuite d'Andalousie, Juan de Sanctivañez parle d' « une nouvelle terre promise » et remarque : «[...] la madrague de Conil se transforma en une gourmandise pour le zèle apostolique de ceux qui ne recherchaient que la plus grande gloire de Dieu et le salut du prochain. Les pères les plus connus, les hommes les plus occupés dans des affaires d'importance demandaient à être envoyés à cette mission ${ }^{49}$. "

Des saisonniers de toute la Péninsule, plus de deux mille personnes, affluent alors sur les terres du Grand d'Espagne. Ces journaliers sont qualifiés de gueux et décrits comme des bandits et des hors-la-loi : « [...] ils commettent mille délits et outrages. Les meurtres, vols, excès en tout genre sont quotidiens », écrit Martin de Roa ${ }^{50}$. Ils constituent une population flottante de déclassés et de pauvres à la recherche de travail : «La période de pêche est leur carême car ce sont des vagabonds qui ne s'arrêtent jamais nulle part et sont toujours à la recherche de leur pauvre pitance », ajoute Roa. Pourtant la note dominante de la documentation sur les missions des madragues est celle du chaos : « [...] lls vivent comme des gens sans loi ni roi », précise Roa; « ils vivent comme des Maures sans roi », note León. La pénétration du milieu des travailleurs est essentielle à la réussite de la mission. En 1557, les deux frères qui officient aux madragues travaillent avec les pêcheurs à remonter les filets. Ils gagnent ainsi l'amitié des journaliers et peuvent entreprendre de les évangéliser en leur enseignant la doctrine chrétienne et en remplaçant leurs chants « indécents " par des cantiques. Les pères Lopez et Mata utilisent les mêmes techniques : soins aux malades, travail dans les madragues. Le père de León, au cours des six missions qu'il effectue à Zahara et Conil, développe l'assistance judiciaire face

49. Juan de Sanctivañez, Historia de la provincia de Andalucía de la Compañía de Jesús por el padre Juan de Sanctivañez de la mesma Comp de IHS y Provincia, BUG, cajas B-48, B-49 et B-50.

50. Martín DE ROA, Historia de la Compañia de Jesús en la provincia de Andalucía, Rome, Bibliotheca Scriptorum Societatis Iesu, 6 G 7, p. 274. 
à la justice expéditive du Duc car les autorités seigneuriales jugent en première instance et les délinquants sont souvent exécutés sans autre forme de procès. Les jésuites obtiennent le bannissement des fauteurs de troubles. Le jésuite écrit : «Depuis que les nôtres se rendent là-bas, les madragues sont tranquilles car nous sommes leurs médiateurs, leurs juges et leurs avocats. »

Les épisodes relatés donnent une représentation des journaliers des pêcheries qui oscille entre le stéréotype propre aux milieux criminels et l'observation sociologique. L'absence d'organisation politique en fait des sauvages et rend impossible un équilibre communautaire. Les rixes pour des questions d'honneur sont nombreuses mais la violence développée par les saisonniers des madragues n'est pas différente de celle relatée dans les archives criminelles; elle est spécifique seulement dans la mesure où elle se déroule dans un cadre transitoire, un no man's land dans lequel la rentabilité économique est primordiale et se heurte souvent aux valeurs, pourtant traditionnelles, des travailleurs de la côte. En effet, si la description du milieu tient quelquefois, dans les chroniques missionnaires, du récit picaresque - León dira qu'il aurait pu tirer un livre de divertissements de sa longue expérience des pêcheries de thon -, elle ne reflète que la distance qui sépare les comportements des normes de l'Église postconciliaire. Ainsi les concubinages traversent les rapports des missionnaires de toute la Péninsule et ne concernent pas seulement les villageois ou les habitants des faubourgs des villes mais aussi les classes dirigeantes. Les jeux de cartes sont un passe-temps courant; leur dénonciation les lie à l'oisiveté vilipendée dans tous les traités sur la pauvreté dans le but de promouvoir le travail comme valeur morale et code de conduite, mais aussi une économie familiale et des loisirs chrétiens. Ainsi les jésuites combattent les jeux de cartes auxquels se livrent les pêcheurs des madragues mais les justifient, s'ils sont modérés, dans la maison ducale car ils sont autorisés pour la noblesse dont le statut interdit toute activité manuelle.

Mais, au-delà des topiques qui caractérisent les couches populaires, la pacification des conflits est une des parties importantes de la mission commanditée par les ducs, propriétaires des madragues. Parfois, les travailleurs se révoltent, cessent le travail, se réfugient armés au sommet d'une dune; ils observent ainsi des sortes de grèves qui paralysent la pêche. Les jésuites s'aventurent alors auprès des mutins qui se sont munis de pierres, les raisonnant pour qu'ils reprennent le travail. D'autres fois, les différends éclatent entre les groupes de journaliers; les missionnaires jouent alors les médiateurs entre les parties pour éviter que le déchaînement de bagarres ne nuise au travail, car les pertes peuvent être très importantes pour le duc de Medina Sidonia. On le voit, ils sont doublement impliqués dans un processus de construction d'un équilibre politique. Ils arbitrent les désaccords entre le Duc et ses employés et les rivalités des journaliers. C'est cette dua- 
lité qui conditionne le succès de la mission et sa continuation. Car il ne s'agit pas seulement de donner par des règles morales une cohésion à un groupe mais aussi d'assurer le respect des hiérarchies sociales et des valeurs qui s'y rattachent.

Les saisonniers des madragues n'ont pas non plus, en raison de leur errance, d'éducation religieuse : « [...] comme ils n'ont pas de domicile et qu'ils n'appartiennent à aucune paroisse, personne ne leur demande de rendre des comptes et beaucoup d'entre eux disent à cor et à cri qu'ils ne se sont pas confessés depuis de nombreuses années ${ }^{51}$. " Tout le travail des missionnaires consistera à les amener, par la prédication l'enseignement de la doctrine chrétienne, à la confession nécessaire pour le salut de leurs âmes et pour leur rédemption sociale. Leur compréhension du milieu est fondamentale, elle est un élément important de la pédagogie missionnaire car elle leur assure le dialogue avec les travailleurs et donc la possibilité de les convertir. Les sermons et confessions s'inscrivent dans un processus de réparation. Ils visent à réintégrer ces hommes dans la communauté chrétienne et à les préparer à assumer une responsabilité dans l'ordre social. Le respect des enseignements qu'ils reçoivent est la garantie d'une stabilité politique. Francisco de Borja écrivait, à propos de la prédication : « Le prédicateur fera bien son métier s'il fixe et fortifie les vérités de la foi catholique [...] Il rappellera l'obéissance que l'on doit aux princes et aux supérieurs ecclésiastiques et laïcs ${ }^{52}$."

Le travail des missionnaires se situe donc à plusieurs niveaux : organisation politique et économique de la communauté des pêcheurs, moralisation des mours, éducation religieuse. La mission vise à articuler le corps social. Elle fournit un patron de comportement social et politique structuré par la pratique religieuse. Ainsi, on passe du chaos initial marqué par les stéréotypes propres aux milieux populaires et aux pauvres, à la construction d'une cité, temporaire ici, où les membres sont des citoyens/fidèles; la caractérisation du milieu, plus convaincante parce que plus proche d'autres réalités sociales connues, acquiert alors des accents plus vraisemblables. Cette association permet le passage du topique à l'élaboration d'un discours missionnaire sur des modèles comportementaux idéaux. Car il est bien évident, comme c'est également le cas des missions rurales, que les acquis sont fragiles et que la documentation d'une mission à l'autre est répétitive. Les réussites fulgurantes rapportées dans ces récits ne viendraient-elles pas confirmer la thèse, défendue par les théoriciens jésuites de

51. P. DE LEÓN, op. cit. supra n. 31, p. 68-69.

52. Francisco DE BORIA, "Tratado breve del modo de predicar el Evangelio », in Vida del santo Padre y gran siervo de Dios el beato Francisco de Borja, Madrid, María de Quiñones, 1644 , p. 472-473. 
la raison d'État ${ }^{53}$, de l'indispensable relation entre politique et religion dans laquelle cette dernière devient un instrument de gouvernement?

L'activité missionnaire développée par les jésuites en Espagne et au Portugal entre 1540 et 1650 , permet de suivre la mise en place et l'évolution d'un ministère qui a occupé nombre de membres de la Compagnie de Jésus et dont l'importance est soulignée par la masse de documents que ces missions intérieures, essentiellement rurales à cette époque, ont produite. Leur caractère quadragésimal leur donne un fort contenu pénitentiel qui souligne la finalité de conversion de cette activité apostolique et commande des stratégies de communication particulièrement visibles dans la prédication. Mais la conversion revêt aussi un sens politique dans la pacification des communautés, notamment dans la pénétration des milieux marginaux comme les saisonniers des madragues de la côte gaditane. La mission intérieure apparaît alors comme un instrument d'articulation du corps social dans le cadre d'une subordination du politique au religieux. L'action missionnaire devient ainsi un mécanisme extraordinaire d'imposition d'une discipline sociale, catholique, fondamentale à l'édifice politique de l'époque moderne.

Marie-Lucie Copete et Federico Palomo (février 1999).

53. Juan DE Mariana, Del rey y de la institución real, 1599, Madrid, M. Rivadeneyra, (Biblioteca de autores españoles [= BAE], vol. XXI), 1950; Pedro DE RIvadeneira, Tratado de la religión y virtudes que debe tener el príncipe cristiano para gobernar y conservar sus estados. Contra lo que Nicolás Maquiavelo y los políticos de estos tiempos enseñan, 1595, BAE, vol. LX. Voir, également, José A. Fernandez-Santamarta, Razón de estado y política en el pensamiento español del barroco (1595-1640), Madrid, Centro de estudios constitucionales, 1996. 